\title{
Physiology and Surgical Correction of the Lower Prognathism (A Review)
}

\author{
by \\ Marsh ROBINSON*
}

\section{Physiological Considerations of Lower Prognathism}

The bony units of the temporomandibular joint are the condylar process of the mandible and the mandibular fossa and articular tubercle of the temporal bone. These 2 bones are not in contact. A specialized disk of connective tissue is interposed, thus forming 2 joints. The temporomeniscus joint has the larger potential joint space, and it is here that there is the ginglymoid type (sliding) motion. In the smaller meniscomandibular joint the arthrodial type (hinge) action occurs. Therefore, we have a hinged joint on a movable or sliding base. Everywhere the head of the condyle goes, the disk goes. A capsular ligament is loosely arranged from the temporal bone to the disk ; this allows for the sliding action. Between the disk and the head of the condyle the attachment is very tight and allows for only the hinged type of action. The bone between the mandibular fossa and the middle fossa of the skull is very thin. The disc is made of specialized connective tissue. It has been said that the disc is irreparable fibrocartilage. In 16 necropsies at Los Angeles County General Hospital it was found that the disc was only fibrous connective tissue. The posterior one-third and the anterior one-sixth of the disc are thick and extremely vascular. The central portion of the disc is avascular. In the vascular portions of the disc there is synovial layer of cells. These cells have the function of secreting synovial fluid to lubricate the joint, supplying nutrient to the central avascular portion, and dissipating any heat which is generated in the joint. This specialized connective tissue between the head of the condyle and the temporal bone is the same type of tissue that is found in the costal-sternal joint and in the radial-carpal joint. These joints are not stress-bearing.

The mandible is often described as a Class III lever. A lever of the third class is one that has a fulcrum at one end and a weight at the other with a force between. It has been natural to classify the mandible as a Class III lever because there is an apparent fulcrum in the temporomandibular joint, and the weight or working area is in the dentures, while the muscle attachment to the mandible is between the dentures and the joint. The powerful elevator muscles of the mandible are inserted approximately halfway between the first molar tooth and the condyle. If the mandible were a lever of the third class, then as much stress would be developed in the temporomandibular joint as in the first molar area. As has been shown, the bone superior to the head of the condyle is very thin. This is a nonstress-bearing type of bone. The disc in

\footnotetext{
* Marsh Robinson, D. D.S., M.D., Professor of Oral Surgery, University of Southern California, U.S.A. The present paper was read before the student body of Nihon University School of Dentistry, November 6, 1963.
} 
this area is vascular and this is a nonstress-bearing type of tissue. There is a synovial layer covering this vascular area of the disc which is also nonstress-bearing. It is inconceivable in reviewing the anatomy and the histology of the temporomandibular joint to believe that this is a stress-bearing joint and that the mandible is a Class III lever.

The nonlever action of the mandible can be explained by a simplified diagram. The temporalis muscle has an average fiber direction of about $45^{\circ}$ from the eye-ear plane in a posterior direction. The masseter and internal pterygoid muscles form a sling at an angle which is approximately bisected by the temporalis muscle. The direction of the fibers of these muscles is approximately from the angle of the jaw to the outer canthus of the eye. When these three muscles act upon the mandible there is a resultant force. This resultant force is in the denture area and leaves the joints free to act as definite guide to mandibular movement. The external pterygoid muscle does not contribute to the closing of the jaw. This muscle, which really consists of two muscles, a sphenomeniscus muscle and a sphenomandibular muscle, is important both in the opening action of the jaws and in the clicking and popping noise heard in the jaws pathologically. Significant stresses in the denture occur only in the isometric contraction of the muscles. In isotonic contraction, or bringing the jaws from a rest position to a tooth contact position, the force is not great. It is only when the teeth are in contact and there is an unchanging dimension between origin and insertion of the muscles of mastication that significant stress in magnitude and duration is developed in the denture and in the muscles.

The muscles of mastication are an antigravity group of muscles. The muscle joint and tendon-sensory nerve involved is the mesencephalic 5th nerve. This is the only sensory nerve whose nucleus is found within the central nervous system. This is the proprioceptive nerve that supplies the muscles of mastication, the temporomandibular joint, and the periodontal ligaments of the teeth. The mandible is the only bone in the body that articulates in 3 places with the same bony unit. It can be seen that, if the articulation of the teeth is not correct, this can be reflected in abnormal muscle action. The joints and their muscles, which work for approximately 15 minutes a day in chewing, may work many hours a day or many hours during the night. Muscle tension develops and interferes with the orderly coordination of the muscle groups. A sliding action develops in the meniscocondylar joint and with discoordination of the muscles the disk precedes or lags behind the condyle in the first part of the opening or closing motion and then snaps into position with an audible noise. Muscle spasm pain develops. At first this is usually unilateral and confined to the muscles of mastication with the commonly encountered symptoms. With time, more groups of muscles become involved, accounting for the more bizarre symptom completes. With a gross sliding action occurring in the inferior joint the vascular supply can be embarrassed because of the perverted position of the disk. This can result in diminution of synovial fluid and initiate degenerative joint changes in the far advanced case.]

\section{Teaching Outlines for Prognathism Surgery at Los Angeles County Hospital}

Teaching a surgical technic is easier if it is standardized. This part of the paper presents an outline of technic for the correction of prognathism by the open oblique 
osteotomy of the ramus. After the operation on the first patient in August, 1954, several modifications were made in the technic. A preliminary report of the first five patients was published. The residents at Los Angeles County Hospital started using this method in October, 1956. Since that time they have performed 78 surgical corrections or an average of 6 patients per resident. Deviation from the standardized technic is not allowed. The residents should learn one technic first and later they can make changes as they desire.

Historical Illustration of Previous Failures
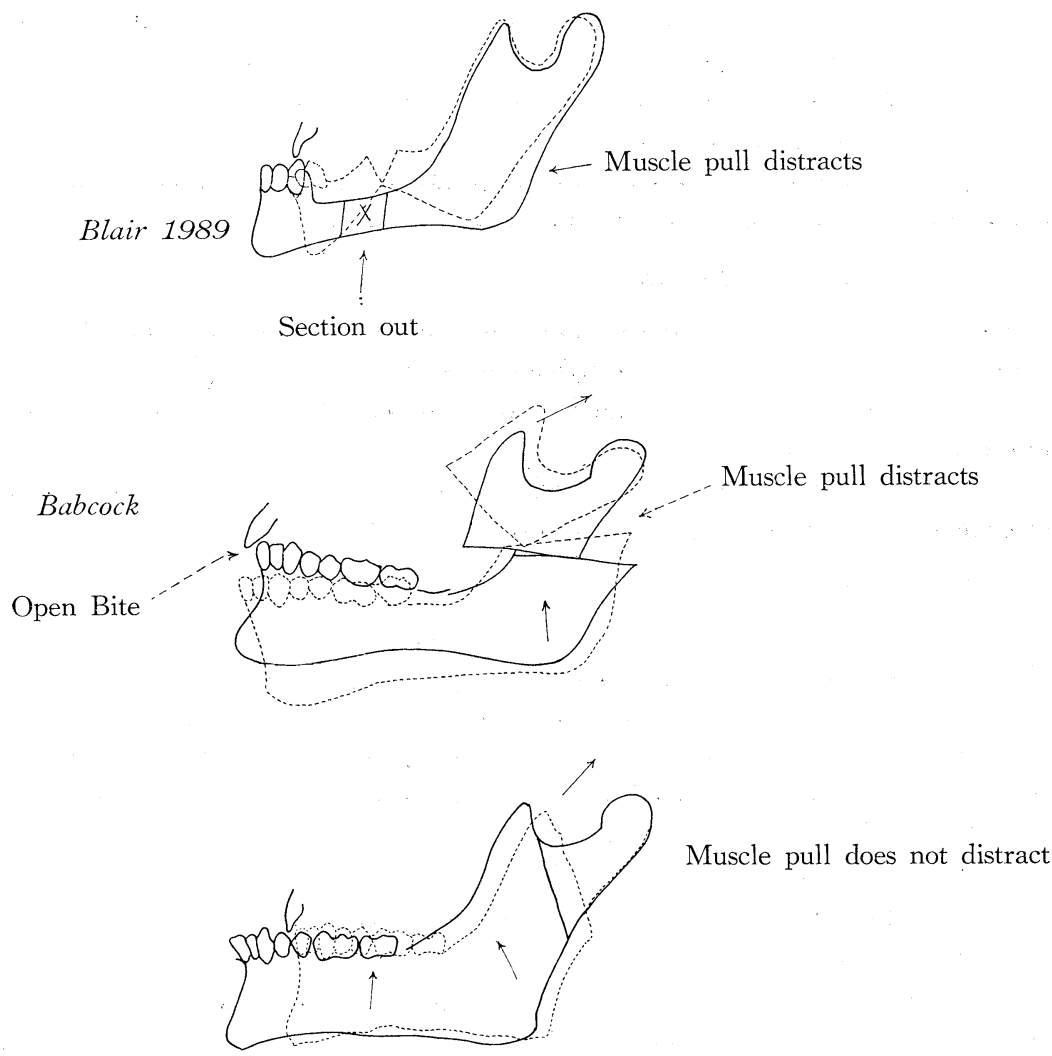

\section{Preoperative Procedures}

1. The intraoral occlusal splint is made and a model is mounted in an overcorrected position from the desired postoperative result. The overcorrection is made by placing the lower model $1.5 \mathrm{~mm}$. in retrusion and opening the bite posteriorly in the second molar area $1 \mathrm{~mm}$.

2. Erich type archbars are placed on the maxillary and mandibular arches of teeth and ligated to each available tooth with .020 stainless steel wire.

3. Any interference in the splint is checked by placing it alternately on each arch.

4. The night before surgery all instruments, headlight, nerve stimulator, and so on, are inspected. 


\section{Surgery}

\section{A. Preparation.}

1. A towel is wrapped about the head, behind the ears, and secured with 1-inch adhesive tape.

2. The position of the patient is considered and a folded sheet placed under the shoulders to extend the head, if it is anticipated that the saw handle will strike the chest.

3. The headlight is adjusted.

4. The Levine and nasotracheal tubes are checked to make sure they are patent and are secured with tape. The tape should not be over the ramus region.

5 . The nurse is instructed to prepare the surgical areas.

6. The surgeon scrubs.

7. A double thickness of towels is placed on a half-sheet under the head. The upper towel is brought over the headcap and secured with clips.

B. Drawing-right side.

1. The ramus is palpated and the outline is drawn on the skin with a sharply broken applicator stick and methylene blue stain.

2. The incision is outlined approximately $4 \mathrm{~cm}$. in length and $1 \mathrm{~cm}$. below the angle in a skin line.

3. The attending surgeon will appraise the incision outline and either make a change or allow the surgery to proceed.

C. Exposure.

1. No. 10 blade is used to incise the skin and subcutaneous tissues. The skin bleeders are clamped with mosquito forceps. No bleeders are tied at this time.

2. The platysma muscle is incised with a No. 15 blade.

3. The tissues are tented beneath the platysma muscle with Adson forceps and strabismus scissors are used for blunt dissection down to the angle. The mandibular branch of the facial nerve is located with the nerve stimulator and retracted upwards.

4. The insertion of the masseter muscle is exposed at the angle.

5. The attending surgeon will palpate the angle and request further dissection to protect the nerve or allow surgery to continue.

6. The insertion of the masseter muscle at the lower border is incised. The periosteum is elevated from the lateral surface of the ramus to a point near the mandibular notch.

7. The mosquito forceps are removed from the skin bleeders. A blunt periosteal elevator is used in the final exposure of the mandibular notch.

D. Osteotomy.

1. The oblique osteotomy site is marked with the saw from a point just posterior to the coronoid process above to the posterior border at some arbitrary point. The mark must be posterior to the inferior dental alveolar nerve and vessels.

2. The attending surgeon will take the retractor and start an incision of the proposed cut. He will modify the direction of the cut, or give the resident the signal to proceed.

3. The cut is made nearly through the bone, with the assistant steadying the 
mandible. Two saws are used alternately with the nurse cleaning the unused saw. If a bleeder is encountered in the center of the bone the assistant should clear the field with suction but the resident should complete the saw cut rapidly.

4. After the saw cut is nearly through the bone, a periosteal elevator is inserted and the proximal fragment is fructured laterally.

5. Pressure is placed on the chin, and the fragments overlapped. The proximal fragment is kept lateral. A 3 by 3 gauze dressing is packed into the wound and the skin closed with a silk suture.

E. Procedures " $B$ " through " $D$ " are repeated on the left side.

F. Intermaxillary fixation.

1. The intraoral splint is placed in position.

2. Five .020 stainless steel intermaxillary wires are twisted around the bars. The lugs on the arch bar are not used.

3. The tongue is moved with a small probe or a bent wire to make sure it is not caught between the teeth and the splint.

4. The attending surgeon will check to see if the tongue is free.

5. Gloves are changed.

G. Closure.

1. Care must be taken that no soft tissue is interposed between the overlapping bone fragments. By the position of the cut it is possible to estimate if there has been any elongation of the ramus to correct an open bite. If there is any component of elongation, then an interosseous wire is placed.

2. The periosteum is closed with two No. 000 chromic gut sutures.

3. A mattress type suture is placed through the platysma muscle in the center of the wound. Two interrupted sutures are placed at either side using No. 0000 chromic gut.

4. The skin is closed with a No. 35 stainless steel subcuticular pullout wire.

\section{H. Bandage.}

The wound is covered with a piece of Telfa dressing over which is placed a 4 gauze. The subcuticular wire is twisted over the gauze. An abdominal dressing is folded one time and placed over each wound. Two four-inch Ace dressings are used to form a bulky collar. The cheeks are coated with tincture of benzein and the collar dressing secured with one inch tape.

\section{Postoperative Procedures}

A. Orders are written and instructions given to the recovery room nurses.

B. The collar is removed after 48 hours.

C. Diet and oral hygiene instructions are given to the patient.

D. Subcuticular wire is removed in seven to ten days.

E. Intermaxillary wires are adjusted weekly and removed at the end of seven weeks.

F. The patient is followed to check the occlusal equilibration and whether other dental care is needed. 